\title{
Top quark pair production via (un)polarized photon collisions in the littlest Higgs model with T-parity at the ILC
}

\author{
Bingfang Yang ${ }^{1,2 *}$ \\ ${ }^{1}$ College of Physics \& Information Engineering, \\ Henan Normal University, Xinxiang 453007, China \\ ${ }^{2}$ Basic Teaching Department, Jiaozuo University, Jiaozuo 454000, China
}

\begin{abstract}
We study the top-quark pair production via polarized and unpolarized photon collisions at the International Linear Collider in the context of the littlest Higgs model with T-parity. We calculate the production cross section of the process $\gamma \gamma \rightarrow t \bar{t}$ and find the effects can be more significant in the -- polarized photon collision mode than in other collision modes, and the relative correction can be expected to reach about $-1 \%$ in the favorable parameter space.
\end{abstract}

PACS numbers: 14.65.Ha,12.15.Lk,12.60.-i,13.85.Lg

*Electronic address: yangbingfang@gmail.com 


\section{INTRODUCTION}

The detailed analysis of the dynamics of top-quark production and decay is a major objective of experiments at the Tevatron, the Large Hadron Collider(LHC), and a possible International Linear Collider(ILC). Top quarks can be produced at hadron colliders via top-antitop pair [1] and single top [2] production channels. For top-pair production, the leading-order (LO) partonic processes are $q \bar{q} \rightarrow t \bar{t}$, which is dominant at Tevatron energies, and $g g \rightarrow t \bar{t}$, dominant at LHC energies. At the ILC, one of the most important reactions will be top-pair production well above the threshold. Because of the small statistics, the top-quark properties have not been precisely measured at the Tevatron. Several tens of millions of top pair signals per year will be produced at the LHC, this large number of top quarks allow very precise measurements in the top-quark properties. Compared to the LHC, the $t \bar{t}$ production cross section is less at the ILC[3], but it will be an ideal place for further investigating the top quark duo to its clean background.

The little Higgs theory constructs the Higgs as a Pseudogoldstone boson to solve the hierarchy problem of the Standard Model(SM)[4]. The littlest Higgs (LH) model [5] is an economical approach to implement this idea, but electroweak precision tests give it strong constraints [6] so that the fine-tuning problem in the Higgs potential would be reintroduced[7]. Then, a discrete symmetry called T-parity is proposed to tackle this problem[8], this resulting model is referred to as the littlest Higgs model with T-parity (LHT).

The LHT model predicts new particles, such as T-odd gauge bosons and T-odd fermions. In the LHT model, there are interactions between the SM fermions and the mirror fermions mediated by the new T-odd gauge bosons or T-odd Goldstone bosons. These interactions can contribute to the $\gamma t \bar{t}$ coupling and the production cross section of the process $\gamma \gamma \rightarrow t \bar{t}$. Furthermore, an additional heavy quark $T^{+}$and its partner $T^{-}$ can also contribute to the $\gamma t \bar{t}$ coupling. In this paper, we will study the polarized and unpolarized $\gamma \gamma$ collisions in the LHT model at the ILC.

This paper is organized as follows. In Sec.II we give a brief review of the LHT model. In Sec.III and IV we respectively calculate the one-loop contributions of the LHT model to the $\gamma \gamma \rightarrow t \bar{t}$ in polarized and unpolarized photon-photon collision modes and show some numerical results at the ILC. Finally, we give our conclusions in Sec.V. 


\section{A BRIEF REVIEW OF THE LHT MODEL}

The LHT model [8] is based on an $S U(5) / S O(5)$ non-linear sigma model, where with the global group $S U(5)$ being spontaneously broken into $S O(5)$ by a $5 \times 5$ symmetric tensor at the scale $f \sim \mathcal{O}(T e V)$, the gauged subgroup $[S U(2) \times U(1)]_{1} \times[S U(2) \times U(1)]_{2}$ of $S U(5)$ is broken to the diagonal subgroup $S U(2)_{L} \times U(1)_{Y}$ of $S O(5)$.

From the symmetry breaking, there arise 4 new heavy gauge bosons $W_{H}^{ \pm}, Z_{H}, A_{H}$ whose masses up to $\mathcal{O}\left(v^{2} / f^{2}\right)$ are given by

$$
M_{W_{H}}=M_{Z_{H}}=g f\left(1-\frac{v^{2}}{8 f^{2}}\right), M_{A_{H}}=\frac{g^{\prime} f}{\sqrt{5}}\left(1-\frac{5 v^{2}}{8 f^{2}}\right)
$$

with $g$ and $g^{\prime}$ being the SM $S U(2)$ and $U(1)$ gauge couplings, respectively.

A consistent viable implementation of T-parity in the fermion sector requires the introduction of mirror fermions. For each SM quark, a copy of mirror quark with T-odd quantum number is added. We denote up-type and down-type mirror quarks by $u_{H}^{i}, d_{H}^{i}$ respectively, where $\mathrm{i}=1,2,3$ are the generation index, whose masses up to $\mathcal{O}\left(v^{2} / f^{2}\right)$ are given by

$$
m_{d_{H}^{i}}=\sqrt{2} \kappa_{i} f, m_{u_{H}^{i}}=m_{d_{H}^{i}}\left(1-\frac{v^{2}}{8 f^{2}}\right)
$$

where $\kappa_{i}$ are the diagonalized Yukawa couplings of the mirror quarks.

An additional heavy quark $T^{+}$is introduced to cancel the large contributions to the Higgs mass from one-loop quadratic divergences. The implementation of T-parity then requires also a T-odd partner $T^{-}$, which is an exact singlet under $S U(2)_{1} \times S U(2)_{2}$. Their masses up to $\mathcal{O}\left(v^{2} / f^{2}\right)$ are given by

$$
\begin{aligned}
& m_{T^{+}}=\frac{f}{v} \frac{m_{t}}{\sqrt{x_{L}\left(1-x_{L}\right)}}\left[1+\frac{v^{2}}{f^{2}}\left(\frac{1}{3}-x_{L}\left(1-x_{L}\right)\right)\right] \\
& m_{T^{-}}=\frac{f}{v} \frac{m_{t}}{\sqrt{x_{L}}}\left[1+\frac{v^{2}}{f^{2}}\left(\frac{1}{3}-\frac{1}{2} x_{L}\left(1-x_{L}\right)\right)\right]
\end{aligned}
$$

where $x_{L}$ is the mixing parameter between the SM top-quark $t$ and the heavy quark $T^{+}$.

In the LHT model, one of the important ingredients of the mirror sector is the existence of four CKM-like unitary mixing matrices, two for mirror quarks and two for mirror leptons:

$$
V_{H u}, V_{H d}, V_{H l}, V_{H \nu}
$$


where $V_{H u}$ and $V_{H d}$ are for the mirror quarks which are present in our analysis. They satisfy the relation $V_{H u}^{\dagger} V_{H d}=V_{C K M}$. We follow Ref.[9] to parameterize $V_{H d}$ with three angles $\theta_{12}^{d}, \theta_{23}^{d}, \theta_{13}^{d}$ and three phases $\delta_{12}^{d}, \delta_{23}^{d}, \delta_{13}^{d}$

$V_{H d}=\left(\begin{array}{ccc}c_{12}^{d} c_{13}^{d} & s_{12}^{d} c_{13}^{d} e^{-i \delta_{12}^{d}} & s_{13}^{d} e^{-i \delta_{13}^{d}} \\ -s_{12}^{d} c_{23}^{d} e^{i \delta_{12}^{d}}-c_{12}^{d} s_{23}^{d} s_{13}^{d} e^{i\left(\delta_{13}^{d}-\delta_{23}^{d}\right)} & c_{12}^{d} c_{23}^{d}-s_{12}^{d} s_{23}^{d} s_{13}^{d} e^{i\left(\delta_{13}^{d}-\delta_{12}^{d}-\delta_{23}^{d}\right)} & s_{23}^{d} c_{13}^{d} e^{-i \delta_{23}^{d}} \\ s_{12}^{d} s_{23}^{d} e^{i\left(\delta_{12}^{d}+\delta_{23}^{d}\right)}-c_{12}^{d} c_{23}^{d} s_{13}^{d} e^{i \delta_{13}^{d}} & -c_{12}^{d} s_{23}^{d} e^{i \delta_{23}^{d}}-s_{12}^{d} c_{23}^{d} s_{13}^{d} e^{i\left(\delta_{13}^{d}-\delta_{12}^{d}\right)} & c_{23}^{d} c_{13}^{d}\end{array}\right)(6)$

\section{TOP QUARK PAIR PRODUCTION VIA $\gamma \gamma$ COLLISION IN THE LHT MODEL}

In the context of the LHT model, the relevant Feynman diagrams of the one-loop correction to the process $\gamma \gamma \rightarrow t \bar{t}$ are shown in Fig.1, where the black dot represents the effective $\gamma t \bar{t}$ vertex which is shown in Fig.2 and the black diamond represents the fermion propagator.

In our calculation, the higher order couplings between the scalar triplet $\Phi$ and top quark and the high order $\mathcal{O}\left(v^{2} / f^{2}\right)$ terms in the masses of new particles and in the Feynman rules are neglected. The relevant Feynman rules can be found in Ref.[10]. We use the 't Hooft-Feynman gauge, so the masses of the Goldstone bosons and the ghost fields are the same as their corresponding gauge bosons. The ultraviolet divergences have been regulated by the dimensional regularization scheme and the divergences have been canceled according to the on-shell renormalization scheme.

For the $\gamma \gamma$ collision at the ILC, the photon beams are generated by the backward Compton scattering of incident electron- and laser-beams just before the interaction point. The total cross section $\sigma(s)$ for the top-pair production can be obtained by folding the elementary cross section $\hat{\sigma}(\hat{s})$ for the subprocess $\gamma \gamma \rightarrow t \bar{t}$ with the photon luminosity at the $e^{+} e^{-}$colliders given in Ref.[11]

$$
\sigma(s)=\int_{2 m_{t} / \sqrt{s}}^{x_{\max }} \mathrm{d} z \frac{\mathrm{d} L_{\gamma \gamma}}{\mathrm{d} z} \hat{\sigma}(\hat{s}) \quad\left(\gamma \gamma \rightarrow t \bar{t} \text { at } \hat{s}=z^{2} s\right),
$$

where $\sqrt{s}$ and $\sqrt{\hat{s}}$ are the $e^{+} e^{-}$and $\gamma \gamma$ center-of-mass energies respectively, and $\mathrm{d} L_{\gamma \gamma} / \mathrm{d} z$ is the photon luminosity, which can be expressed as

$$
\frac{\mathrm{d} L_{\gamma \gamma}}{\mathrm{d} z}=2 z \int_{z^{2} / x_{\max }}^{x_{\max }} \frac{\mathrm{d} x}{x} F_{\gamma / e}(x) F_{\gamma / e}\left(z^{2} / x\right) .
$$




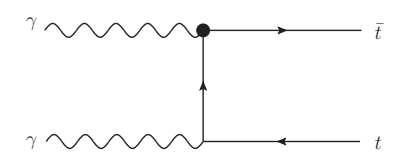

(a)

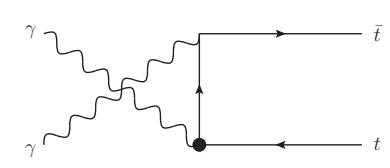

(d)

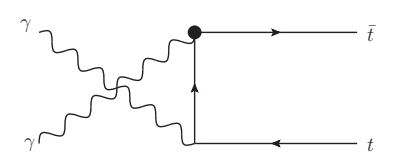

(b)

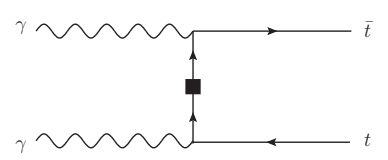

(e)

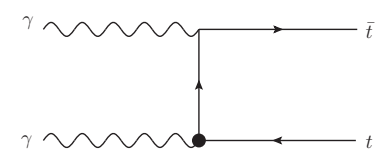

(c)

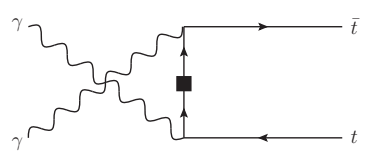

(f)

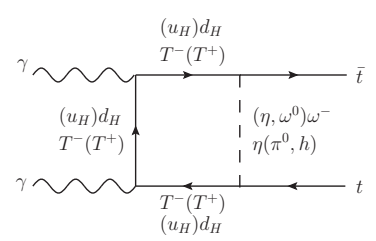

(g)

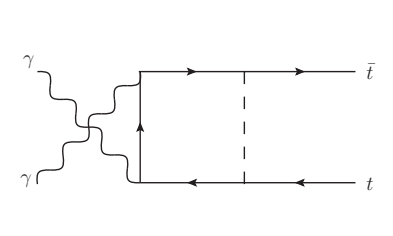

(h)

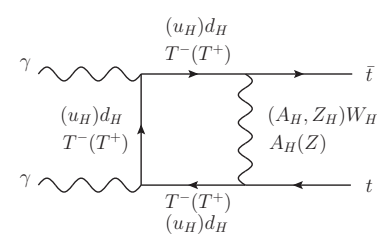

(i)

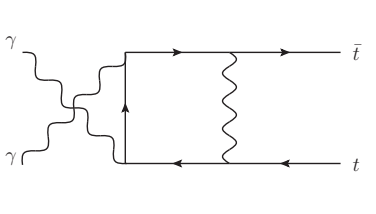

(j)

FIG. 1: Feynman diagrams of the one-loop correction to the process $\gamma \gamma \rightarrow t \bar{t}$ in the LHT model.
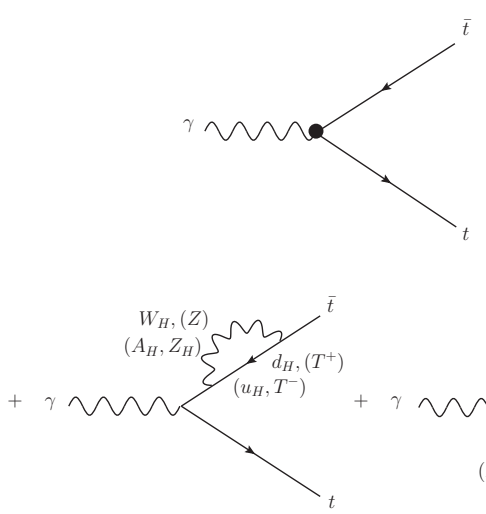

(c)

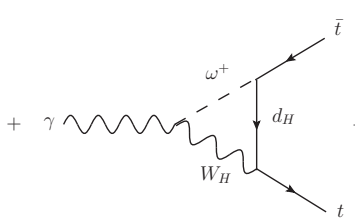

$(g)$

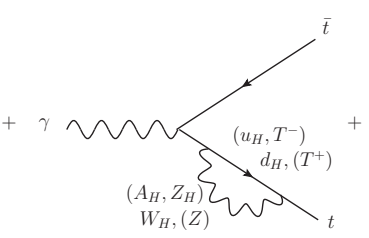

$(d)$

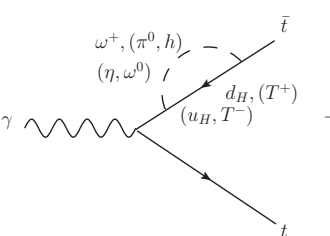

(a)

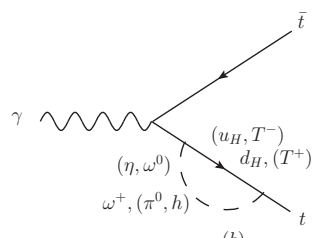

(b)

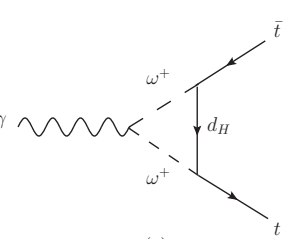

$(e)$

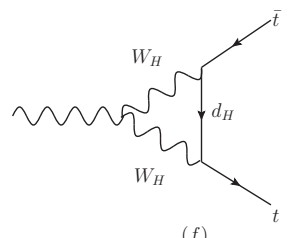

$(f)$

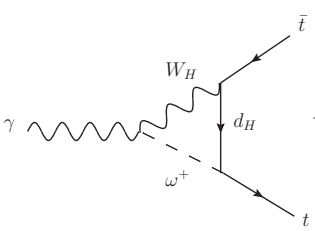

(h)

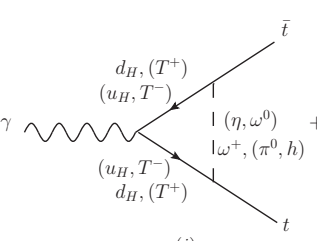

(i)

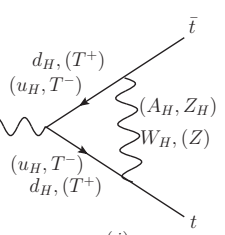

$(j)$

FIG. 2: The effective $\gamma t \bar{t}$ vertex diagrams at one-loop level in the LHT model.

For unpolarized initial electron and laser beams, the energy spectrum of the backscattered photon is given by

$$
F_{\gamma / e}(x)=\frac{1}{D(\xi)}\left(1-x+\frac{1}{1-x}-\frac{4 x}{\xi(1-x)}+\frac{4 x^{2}}{\xi^{2}(1-x)^{2}}\right) .
$$

where $\xi=4 E_{e} E_{0} / m_{e}^{2}, m_{e}$ and $E_{e}$ are respectively the incident electron mass and energy, 
$E_{0}$ is the initial laser photon energy. In our numerical calculation, we choose $\xi=4.8$, $D(\xi)=1.83$ and $x_{\max }=0.83$.

The $\gamma \gamma$ collisions have five polarization modes as follows:,,,++--+--+ and unpolarized collision modes, where the notation + and - represent the helicities of the two incoming photons being $\lambda_{1}=1$ and $\lambda_{1}=-1$, respectively.

\section{NUMERICAL RESULTS}

In our numerical calculations, we take the SM parameters as [12]

$$
\begin{aligned}
G_{F} & =1.16637 \times 10^{-5} \mathrm{GeV}^{-2}, \quad S_{W}^{2}=\sin ^{2} \theta_{W}=0.231, \\
\alpha_{e} & =1 / 128, M_{Z_{L}}=91.2 \mathrm{GeV}, m_{t}=172.4 \mathrm{GeV}, m_{h}=120 \mathrm{GeV} .
\end{aligned}
$$

The LHT parameters relevant to our study are the scale $f$, the mixing parameter $x_{L}$, the mirror quark masses and the parameters in the matrices $V_{H u}, V_{H d}$. For the mirror quark masses, we get $m_{u_{H}^{i}}=m_{d_{H}^{i}}$ at $\mathcal{O}(v / f)$ and further assume

$$
m_{u_{H}^{1}}=m_{u_{H}^{2}}=m_{d_{H}^{1}}=m_{d_{H}^{2}}=M_{12}, m_{u_{H}^{3}}=m_{d_{H}^{3}}=M_{3}
$$

From the couplings between the mirror quarks and the heavy gauge bosons or the heavy Goldstone bosons, we can see the main contribution comes from the third family couplings. In order to show the largest correction, for the matrices $V_{H u}, V_{H d}$, we follow Ref.[13] to choose the following scenario: $V_{H u}=1, V_{H d}=V_{C K M}$. In this scenario, the contribution of the LHT model comes entirely from the third family mirror quarks and the additional heavy quarks $T^{+}, T^{-}$.

In Fig.3(a), we discuss the dependance of the relative correction $\delta \sigma / \sigma$ on the centerof-mass energy $\sqrt{s}$. To see the influence of the scale $f$ on the $\delta \sigma / \sigma$, we take $f=$ $500,2000 \mathrm{GeV}$, respectively. We can see $\delta \sigma / \sigma$ firstly decreases then increases with $\sqrt{s}$. The higher the scale $f$ is taken, the shallower the curve becomes. To see the influence of the $x_{L}$ on the $\delta \sigma / \sigma$, we take $x_{L}=0.1,0.7$, respectively. We can see that the larger the $x_{L}$ is taken, the less the negative relative correction is generated. Furthermore, the larger the scale $f$ is taken, the less influence the $x_{L}$ can exert, which means the contributions of the $T^{+}, T^{-}$are supressed by the high scale $f$. Considering the constraints of Ref.[14], the maximum of the relative correction can reach about $-0.65 \%$. 


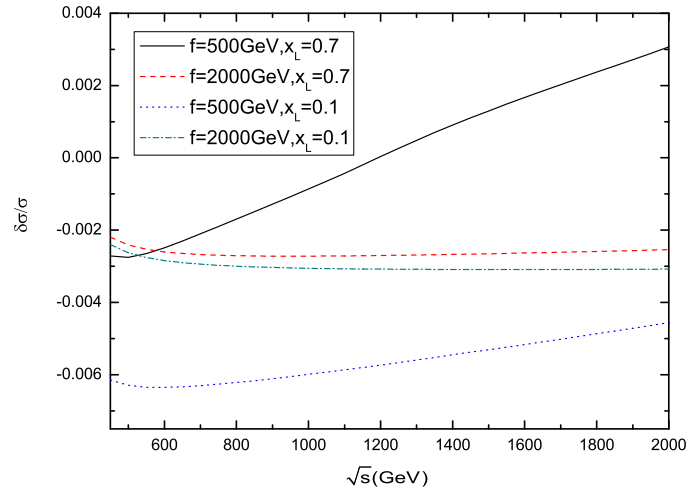

(a)

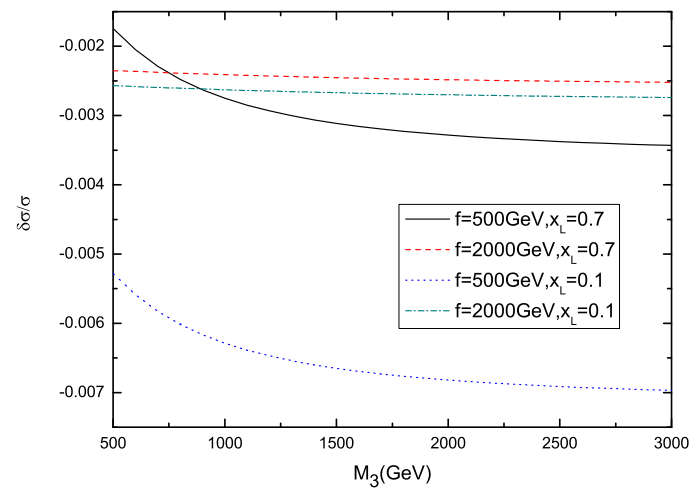

(b)

FIG. 3: The relative correction of the top-quark pair production cross section $\delta \sigma / \sigma$ as functions of the center-of-mass energy $\sqrt{s}$ for $M_{3}=1000 \mathrm{GeV}(\mathrm{a})$ and the mirror quark mass $M_{3}$ for $\sqrt{s}=500 G e V(\mathrm{~b})$ in unpolarized photon collision mode, respectively.

In Fig.3(b), we discuss the dependance of $\delta \sigma / \sigma$ on the third family mirror quark mass $M_{3}$. We can see $\delta \sigma / \sigma$ is negative and becomes larger with the $M_{3}$ increasing. Same as above, we take $f=500,2000 \mathrm{GeV}$ respectively to see the influence of the scale $f$ on the $\delta \sigma / \sigma$ and take $x_{L}=0.1,0.7$ respectively to see the influence of the $x_{L}$ on the $\delta \sigma / \sigma$. The larger the scale $f$ is taken, the shallower the curve becomes, which means the contributions of the $T^{+}, T^{-}$and the third family mirror quark are all supressed by the high scale $f$. For the same $x_{L}$, the overall trend is that the higher the scale $f$ is taken, the smaller the relative correction $\delta \sigma / \sigma$ is generated. For the same scale $f$, the larger the $x_{L}$ is taken, the less the relative correction $\delta \sigma / \sigma$ is generated, which means $\delta \sigma / \sigma$ becomes less with the $M_{T^{+}}, M_{T^{-}}$increasing. Furthermore, we find the contribution of the third family mirror quark is negative while the contributions of the $T^{+}, T^{-}$change from negative to positive with the $x_{L}$ from 0.1 to 0.7 . For the case $f=500 \mathrm{GeV}, x_{L}=0.7$, the contributions of the $T^{+}, T^{-}$are positive so that they counteract the contribution of the third family mirror quark very strongly. As a result, the $\delta \sigma / \sigma$ for $f=500 G e V, x_{L}=0.7$ is smaller than the case for $f=2000 \mathrm{GeV}, x_{L}=0.7$ when $M_{3}<750 \mathrm{GeV}$. For the case $f=500 \mathrm{GeV}, x_{L}=0.1$, the contributions of the $T^{+}, T^{-}$are negative so that the contribution of the third family mirror quark is enhanced obviously. The maximum of the relative correction can reach about $-0.7 \%$. 


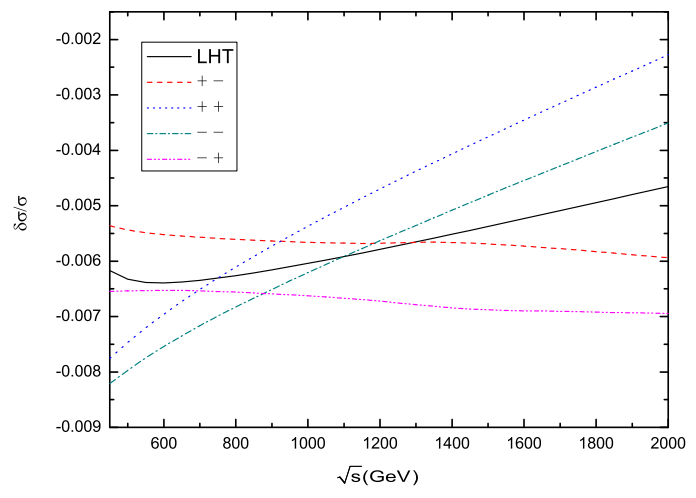

(a)

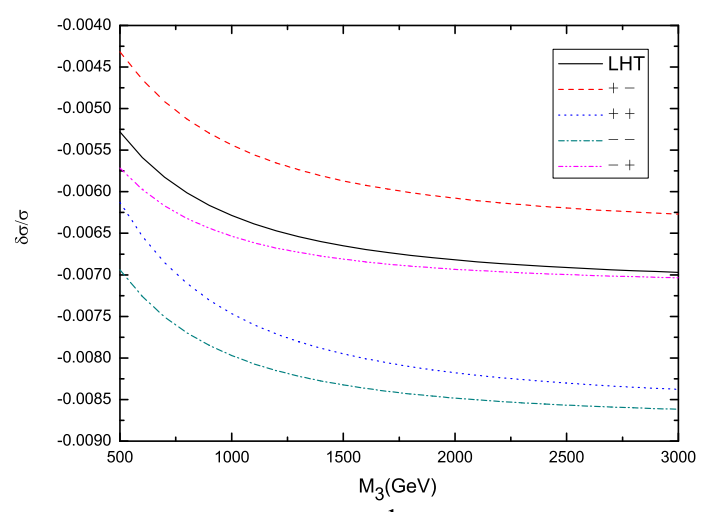

(b)

FIG. 4: The relative correction of the top-quark pair production cross section $\delta \sigma / \sigma$ as functions of the center-of-mass energy $\sqrt{s}$ for $M_{3}=1000 \mathrm{GeV}, f=500 \mathrm{GeV}, x_{L}=0.1$ (a) and the mirror quark mass $M_{3}$ for $\sqrt{s}=500 G e V, f=500 G e V, x_{L}=0.1(\mathrm{~b})$, respectively.

To see the maximum of the relative correction in the LHT model, we take $f=$ $500 \mathrm{GeV}, x_{L}=0.1$ for the process $\gamma \gamma \rightarrow t \bar{t}$ in polarized photon collision mode. We show the dependance of the relative correction $\delta \sigma / \sigma$ on the center-of-mass energy $\sqrt{s}$ and the third family mirror quark mass $M_{3}$ for the process $\gamma \gamma \rightarrow t \bar{t}$ with unpolarized and completely,,,+-++---+ polarized photon beams in Fig4.(a) and Fig4.(b), respectively. From Fig.4(a) and Fig.4(b) we can see clearly that the relative correction $\delta \sigma / \sigma$ in -photon polarization collision mode are larger than in other photon collision modes. In -- photon polarization collision mode, the maximum of the relative correction can be expected to reach about $-1 \%$ in the favorable parameter space.

\section{CONCLUSIONS}

In the framework of the LHT model, we studied the one-loop contributions of the Todd particles to the top-quark pair production cross section in unpolarized and polarized photon collision modes. Because the contributions of the $T^{+}, T^{-}$change from negative to positive with the $x_{L}$ increasing, in some cases the contribution of the third family mirror quark to the relative correction $\delta \sigma / \sigma$ was enhanced, and in other cases the contribution was counteracted. In all collision modes, we found that the relative correction $\delta \sigma / \sigma$ can be more significant in the -- polarized photon collision mode than in other collision 
modes. In the favorable parameter space, the relative correction can be expected to reach about $-1 \%$.

\section{Acknowledgments}

We thank Cao Jun-jie for providing the calculation programs and thank Wu Lei for useful discussions. This work is supported by the National Natural Science Foundation of China under Grant Nos.10775039, 11075045, by Specialized Research Fund for the Doctoral Program of Higher Education under Grant No.20094104110001 and by HASTIT under Grant No.2009HASTIT004.

Appendix: The expression of the renormalization vertex $\hat{\Gamma}_{\gamma t \bar{t}}^{\mu}$ and the renormalization propagator $-i \hat{\Sigma}^{f}(p)[15]$

(I)Renormalization vertex

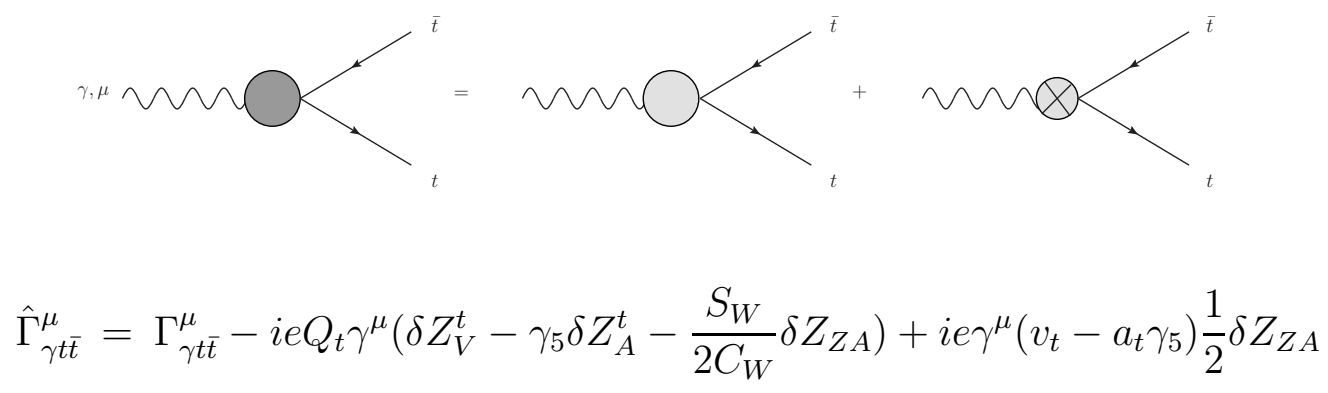

where

$$
\begin{aligned}
v_{t} & \equiv \frac{I_{t}^{3}-2 Q_{t} S_{W}^{2}}{2 C_{W} S_{W}}, \quad a_{t} \equiv \frac{I_{t}^{3}}{2 C_{W} S_{W}}, \quad I_{t}^{3}=\frac{1}{2}, \quad Q_{t}=\frac{2}{3} \\
\delta Z_{Z A}= & 2 \frac{\Sigma_{T}^{A Z}(0)}{M_{Z_{L}}^{2}} \\
\delta Z_{L}^{t}= & \operatorname{Re} \Sigma_{L}^{t}\left(m_{t}^{2}\right)+\left.m_{t}^{2} \frac{\partial}{\partial P_{t}^{2}} \operatorname{Re}\left[\Sigma_{L}^{t}\left(P_{t}^{2}\right)+\Sigma_{R}^{t}\left(P_{t}^{2}\right)+2 \Sigma_{S}^{t}\left(P_{t}^{2}\right)\right]\right|_{P_{t}^{2}=m_{t}^{2}} \\
\delta Z_{R}^{t}= & \operatorname{Re} \Sigma_{R}^{t}\left(m_{t}^{2}\right)+\left.m_{t}^{2} \frac{\partial}{\partial P_{t}^{2}} \operatorname{Re}\left[\Sigma_{L}^{t}\left(P_{t}^{2}\right)+\Sigma_{R}^{t}\left(P_{t}^{2}\right)+2 \Sigma_{S}^{t}\left(P_{t}^{2}\right)\right]\right|_{P_{t}^{2}=m_{t}^{2}} \\
\delta Z_{V}^{t}= & \frac{1}{2}\left(\delta Z_{L}^{t}+\delta Z_{R}^{t}\right), \delta Z_{A}^{t}=\frac{1}{2}\left(\delta Z_{L}^{t}-\delta Z_{R}^{t}\right) \\
\hat{\Gamma}_{\gamma H \bar{t}}^{L H, \mu} & =\Gamma_{\gamma t \bar{t}}^{\mu}(\eta)+\Gamma_{\gamma t \bar{t}}^{\mu}\left(\omega^{0}\right)+\Gamma_{\gamma t \bar{t}}^{\mu}\left(\omega^{ \pm}\right)+\Gamma_{\gamma t \bar{t}}^{\mu}\left(\pi^{0}\right)+\Gamma_{\gamma t \bar{t}}^{\mu}(h) \\
& +\Gamma_{\gamma t \bar{t}}^{\mu}\left(A_{H}\right)+\Gamma_{\gamma t \bar{t}}^{\mu}\left(Z_{H}\right)+\Gamma_{\gamma t \bar{t}}^{\mu}\left(W_{H}^{ \pm}\right)+\Gamma_{\gamma t \bar{t}}^{\mu}(Z)+\Gamma_{\gamma t \bar{t}}^{\mu}\left(\omega^{ \pm}, W_{H}^{ \pm}\right) \\
& +\delta \Gamma_{\gamma t \bar{t}}^{\mu}(\eta)+\delta \Gamma_{\gamma t \bar{t}}^{\mu}\left(\omega^{0}\right)+\delta \Gamma_{\gamma t \bar{t}}^{\mu}\left(\omega^{ \pm}\right)+\delta \Gamma_{\gamma t \bar{t}}^{\mu}\left(\pi^{0}\right)+\delta \Gamma_{\gamma t \bar{t}}^{\mu}(h) \\
& +\delta \Gamma_{\gamma t \bar{t}}^{\mu}\left(A_{H}\right)+\delta \Gamma_{\gamma t \bar{t}}^{\mu}\left(Z_{H}\right)+\delta \Gamma_{\gamma t \bar{t}}^{\mu}\left(W_{H}^{ \pm}\right)+\delta \Gamma_{\gamma t \bar{t}}^{\mu}(Z)
\end{aligned}
$$


(II)Renormalization propagator

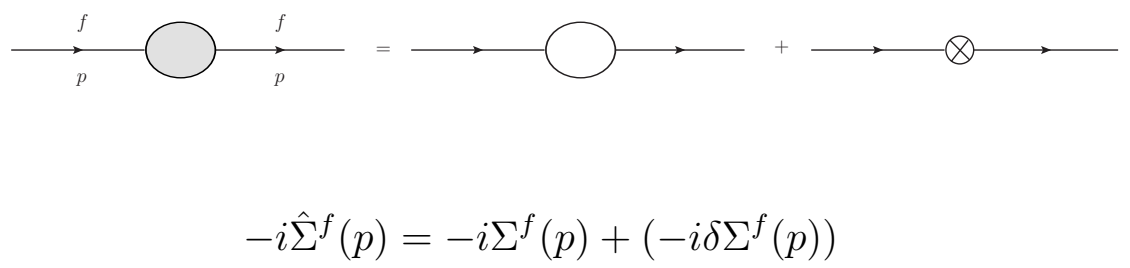

where

$$
\begin{aligned}
\Sigma^{f}(p) & =m_{f} \Sigma_{S}^{f}\left(p^{2}\right)+\not p P_{L} \Sigma_{L}^{f}\left(p^{2}\right)+\not p P_{R} \Sigma_{R}^{f}\left(p^{2}\right) \\
\delta \Sigma^{f}(p) & =\delta m_{f}+m_{f} \frac{1}{2} \delta Z_{L}^{f}+m_{f} \frac{1}{2} \delta Z_{R}^{f}-\not p P_{L} \delta Z_{L}^{f}-\not p P_{R} \delta Z_{R}^{f} \\
\delta m_{f} & =-m_{f} \operatorname{Re}\left[\Sigma_{S}^{f}\left(m_{f}^{2}\right)+\frac{1}{2} \Sigma_{L}^{f}\left(m_{f}^{2}\right)+\frac{1}{2} \Sigma_{R}^{f}\left(m_{f}^{2}\right)\right] \\
\delta Z_{L}^{f} & =\operatorname{Re} \Sigma_{L}^{f}\left(m_{f}^{2}\right)+\left.m_{f}^{2} \frac{\partial}{\partial p^{2}} \operatorname{Re}\left[\Sigma_{L}^{f}\left(p^{2}\right)+\Sigma_{R}^{f}\left(p^{2}\right)+2 \Sigma_{S}^{f}\left(p^{2}\right)\right]\right|_{p^{2}=m_{f}^{2}} \\
\delta Z_{R}^{f} & =\operatorname{Re} \Sigma_{R}^{f}\left(m_{f}^{2}\right)+\left.m_{f}^{2} \frac{\partial}{\partial p^{2}} \operatorname{Re}\left[\Sigma_{L}^{f}\left(p^{2}\right)+\Sigma_{R}^{f}\left(p^{2}\right)+2 \Sigma_{S}^{f}\left(p^{2}\right)\right]\right|_{p^{2}=m_{f}^{2}}
\end{aligned}
$$

[1] CDF Coll., F. Abe et al., Observation of Top Quark Production in Pbar-P Collisions with the Collider Detector at Fermilab, Phys. Rev. Lett. 74 (1995) 2626 hep-ex/9503002; D0 Coll., S. Abachi et al., Observation of the Top Quark, Phys. Rev. Lett. 74 (1995) 2632 hep-ex/9503003.

[2] D0 Coll., V.M. Abazov et al., Observation of Single Top-Quark Production, Phys. Rev. Lett. 103 (2009) 092001, arXiv:0903.0850 [hep-ex]; CDF Coll., T. Aaltonen et al., Observation of Electroweak Single Top-Quark Production, Phys. Rev. Lett. 103 (2009) 092002, arXiv:0903.0885 [hep-ex].

[3] G. Weiglein et al. [LHC/LC Study Group], Phys. Rept. 426 (2006) 47 arXiv:hep-ph/0410364; J. A. Aguilar-Saavedra et al. [ECFA/DESY LC Physics Working Group], arXiv:hep-ph/0106315.

[4] N. Arkani-Hamed, A. G. Cohen, and H. Georgi, Phys. Lett. B 513 (2001) 232; N. ArkaniHamed, et al., JHEP 0208 (2002) 020; JHEP 0208 (2002) 021; I. Low, W. Skiba, and D.Smith, Phys. Rev. D 66 (2002) 072001; D. E. Kaplan and M. Schmaltz, JHEP 0310 (2003) 039 . 
[5] N. Arkani-Hamed, A. G. Cohen, E. Katz, and A. E. Nelson, JHEP 0207 (2002) 034; S.Chang, JHEP 0312 (2003) 057; T. Han, H. E. Logan, B. McElrath, and L. T. Wang, Phys.Rev. D 67 (2003) 095004; M. Schmaltz and D. Tucker-smith, Ann. Rev. Nucl. Part. Sci. 55 (2005) 229.

[6] C.Csaki, J.Hubisz, G.D.Kribs, P.Meade, J.Terning, Phys. Rev. D 67 (2003) 115002; Phys. Rev. D 68 (2003) 035009; J. L. Hewett,F. J. Petriello, and T. G. Rizzo, JHEP 0310 (2003) 062; M. C. Chen and S. Dawson, Phys.Rev. D 70 (2004) 015003; M. C. Chen, et al., Mod. Phys. Lett. A 21 (2006) 621; W. Kilian, J. Reuter, Phys. Rev. D 70 (2004) 015004.

[7] G. Marandella, C. Schappacher and A. Strumia, Phys. Rev. D 72 (2005) 035014.

[8] H. C. Cheng and I. Low, JHEP 0309 (2003) 051; JHEP 0408 (2004) 061; I. Low, JHEP 0410 (2004) 067; J. Hubisz and P. Meade, Phys. Rev. D 71 (2005) 035016.

[9] M.Blanke, et al., Phys. Lett. B 646 (2007) 253.

[10] M.Blanke, et al., JHEP 0701 (2007) 066.

[11] I. F. Ginzburg et al., Nucl. Instrum. 219 (1984) 5; V. I. Telnov, Nucl. Instrum. Meth. 294 (1990) 72 .

[12] C. Amsler, et al., Phys. Lett. B 667 (2008) 1.

[13] M.Blanke, et al., JHEP 0705 (2007) 013.

[14] J. Hubisz, P. Meade, A. Noble, and M. Perelstein, JHEP 0601 (2006) 135; Bingfang Yang, Xuelei Wang, and Jinzhong Han, Nucl. Phys. B 847 (2011) 1.

[15] W.F.L.Hollik, Fortschr. Phys. 38 (1990) 165-260; A.Denner, Fortschr. Phys.41 (1993) 307420. 